\title{
Direct Measurement of the Differential Capacitance of Solvent-Free and Dilute Ionic Liquids
}

\author{
Monchai Jitvisate ${ }^{\dagger}$ and James R. T. Seddon* $*$ *0 \\ ${ }^{\dagger}$ Nanoionics, MESA+ Institute for Nanotechnology, University of Twente, P.O. Box 217, 7500 AE Enschede, The Netherlands \\ ${ }^{\ddagger}$ Physics of Complex Fluids, MESA+ Institute for Nanotechnology, University of Twente, P.O. Box 217, 7500 AE Enschede, The \\ Netherlands
}

\section{Supporting Information}

ABSTRACT: Differential capacitance is a key quantity in the understanding of electrical double-layer charging of electrolytes. However, experimental observations of ionic liquid systems are controversial, inconsistent, and often unable of confirming or refuting existing theories as well as highlighting discrepancies between the measurement techniques. We study the differential capacitance in both pure and dilute ionic liquids at room temperature. Using chronoamperometry to measure the differential capacitance of the liquids at a polycrystalline platinum electrode, we find good agreement between the measured capacitance curves and the extended mean-field model of GoodwinKornyshev [Goodwin, Z. A.; et al. Electrochim. Acta. 2017, 225, 190-197]. A crossover is found from the pure to the dilute regime, as shown by a transition from a camel-shape capacitance curve to a U-like one, together with a nonmonotonic dependence of capacitance with electrolyte concentration.

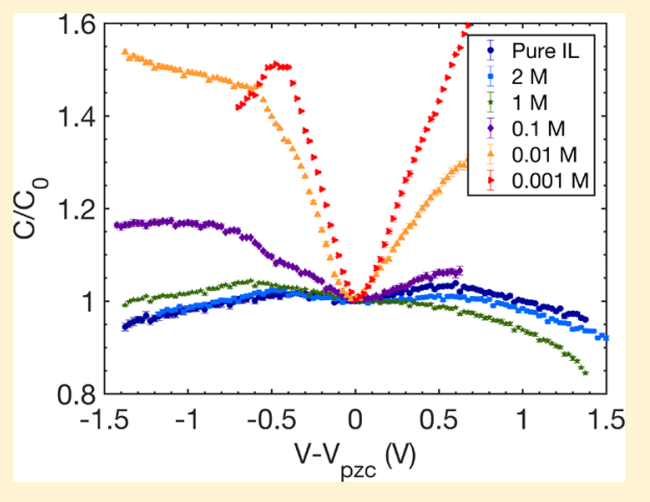

$\mathrm{R}$ oom-temperature ionic liquids have drawn huge attention recently due to their significant promise for many applications, such as electrochemical energy-storage devices, electrically tunable lubrication, high-temperature/vacuum material synthesis, and novel self-assembly media. ${ }^{1-4}$ The reason for excitement stems from their material properties, which are of technological interest. For example, ionic liquids have wide electrochemical window (ECW), high ionic strength, high temperature stability, and low vapor pressure. ${ }^{2,5,6}$ The development of ionic-liquid-based energy devices, such as supercapacitors, requires an insightful understanding of the electrical double-layer (EDL) charging mechanism, for which the traditional (Gouy-Chapman) model appears inappropriate. $^{6-14}$ An important quantity related to EDL charging is the differential capacitance, which represents the variation of the surface charge with respect to the change of surface potential. It is one of the key properties intensively studied in the field of ionic liquids, from theoretical, numerical, and experimental aspects. $^{6-32}$ However, experimentally measuring the differential capacitance of ionic liquids has proven to be a great challenge. $6,8-12,19-26$

Previous studies have focused on two techniques-cyclic voltammetry $(\mathrm{CV})$ and electrochemical impedance spectroscopy (EIS) - showing that the liquids tend to exhibit complex responses due to their large, bulky molecular dimensions. However, the data from these experiments are inconsistent and often irreproducible. ${ }^{6,8-12,19-26,30-32}$ In both cases, the techniques themselves are most likely the causes of discrepancies, for example, the assumption of negligible time dependence of capacitances in $\mathrm{CV}$ and the validity of the equivalent circuit model and relevant time scale in EIS. ${ }^{10,11,28,29,33-37}$ Many EIS works have even adopted a single-frequency method, even though it is known that a fullfrequency spectrum should be recorded. ${ }^{8,9,19-21,23-25}$ In short, neither of these techniques provides a direct measurement of the differential capacitance of the ionic liquids, and this disagreement has resulted in inconsistent and confusing messages about the true nature of ionic liquids at electrified electrodes, holding back theoretical advancement.

In this study, we measure the differential capacitance of ionic liquids using the chronoamperometry (CA) technique, which allows direct charge measurement and direct calculation of differential capacitance. To the best of our knowledge, this is the first application to ionic liquids. This technique thus gives us experimental access to the differential capacitance without the need for unsupported or questionable assumptions. We find good agreement with the mean-field model of GoodwinKornyshev, ${ }^{13,14}$ which is based on that of Kornyshev, ${ }^{7}$ extended by adding short-range ion correlations. Our results include a transition of capacitance curves from camel shape to U-like shape as the liquid is diluted, which is important for understanding the nature of ionic liquids and developing real applications, where the capacitance and other quantities, such as, electrode potential, viscosity, liquid volume, and so on, need to be optimized.

Received: November 6, 2017

Accepted: December 19, 2017

Published: December 19, 2017 



Figure 1. (a) Example of step-function potential and corresponding measured charging current. (b) Potential-dependent charge density of pure ionic liquids $\left[\mathrm{C}_{4} \mathrm{mpyr}\right]^{+}\left[\mathrm{NTf}_{2}\right]^{-},[\mathrm{Emim}]^{+}\left[\mathrm{NTf}_{2}\right]^{-},[\mathrm{Emim}]^{+}\left[\mathrm{BF}_{4}\right]^{-}$, and $[\mathrm{Emim}]^{+}[\mathrm{DCA}]^{-}$determined from the charging current.

The ionic liquids 1-ethyl-3-methylimidazolium tetrafluoroborate $\left([\mathrm{Emim}]^{+}\left[\mathrm{BF}_{4}\right]^{-}\right)$(Sigma-Aldrich), 1-ethyl-3-methylimidazolium dicyanamide $\left([\text { Emim }]^{+}[\mathrm{DCA}]^{-}\right)$, 1-ethyl-3methylimidazolium bis(trifluoromethylsulfonyl)imide $\left([\text { Emim }]^{+}\left[\mathrm{NTf}_{2}\right]^{-}\right)$, and 1-buthyl-1-methylpyrrolidinium bis(trifluoromethylsulfonyl)imide $\left(\left[\mathrm{C}_{4} \mathrm{mpyr}\right]^{+}\left[\mathrm{NTf}_{2}\right]^{-}\right)$(Merck Millipore) as well as the solvent dimethyl sulfoxide (SigmaAldrich) were purchased with purity of $>99 \%$. They were further purified at $150{ }^{\circ} \mathrm{C}$ under vacuum for $>12 \mathrm{~h}$. In this study, the measurements were done using a standard threeelectrode system. A $2 \mathrm{~mm}$ diameter polycrystalline platinum working electrode (BASi) was cleaned by polishing on a polishing cloth (BUEHLER micro cloth) with $0.3 \mu \mathrm{m}$ alumina paste (BUEHLER Alpha micropolish II), rinsed with Milli-Q water for several minutes, and dried with nitrogen gas. The coiled platinum pseudoreference electrode and counter electrode ( $>99.99 \%$, Sigma-Aldrich), with surface areas greater than the working electrode, were cleaned by flaming in a hot butane flame until glowing red and were immediately placed in cool Milli-Q water, followed by drying with nitrogen gas. All electrodes and glass containers were cleaned before each single measurement. The volume of the test liquid in each measurement was $500 \mu \mathrm{L}$.

$\mathrm{CV}$ and CA were performed using a computer-controlled potentiostat (CH-Instrument, CHI832B). Cyclic voltammograms were recorded separately before the CA measurements for electrochemical window evaluation (Supporting Information). The open-circuit potential (OCP) was measured and found to have values close to $0 \mathrm{~V}$ for all pure ionic liquids but shifted when solvent was added. These OCPs were chosen to be the initial potentials for CA measurements, as suggested in the literature for high reproducibility. ${ }^{8}$ The initial potential can lead to a hysteresis effect and irreproducible results, as shown in the Supporting Information.

We applied a step-function potential of $25 \mathrm{mV} /$ step $\left(\sim k_{\mathrm{B}} T\right)$ to a polycrystalline platinum electrode and measured the current density as a function of time. For each potential step, the voltage was stepped back and forth 10 times between lower and higher potentials to minimize hysteresis effects. ${ }^{38}$ The time duration for each step was $100 \mathrm{~ms}$, which was found to be long enough for the current to reach its steady state (Supporting Information). The potential scan was terminated when it reached the ECW limits. Then, all of the electrodes and the container were cleaned and fresh liquid was replaced for the new measurement in the opposite scan direction. The current was measured with the sampling rate of $0.05 \mathrm{~ms}$ and the sensitivity of $10^{-5} \mathrm{~A} / \mathrm{V}$. All chemical preparation and measurements were carried out at $25{ }^{\circ} \mathrm{C}$ in an MBruan
LABmaster glovebox filled with a nitrogen atmosphere (water and oxygen $<0.1 \mathrm{ppm}$ ). In total, more than 1000 data set were recorded for the present work.

We first present the measurement of differential capacitance in pure ionic liquids $[\mathrm{Emim}]^{+}\left[\mathrm{BF}_{4}\right]^{-},[\mathrm{Emim}]^{+}[\mathrm{DCA}]^{-}$, $[\text { Emim }]^{+}\left[\mathrm{NTf}_{2}\right]^{-}$, and $\left[\mathrm{C}_{4} \mathrm{mpyr}\right]^{+}\left[\mathrm{NTf}_{2}\right]^{-}$, which will allow comparison between our results and those reported in the literature and the validation of our method and data with the theoretical model of Goodwin-Kornyshev. ${ }^{13,14}$ An example of the potential waveform and the corresponding measured current is shown in Figure 1a. The current shows excellent agreement when fitted with the exponential equation, $j=a \exp$ $(-t / \tau)$, where $j$ is the current density and $t$ is time (Supporting Information). The fitting parameters $a$ and $\tau$ are used for the calculation of the charge density. It is clear that the current reaches its equilibrium within the measured time duration, with the exponential fit indicating that the charging mechanism obeys a normal diffusive charging process. ${ }^{39}$ In principle, $\tau$ represents the characteristic charging time constant, which is defined as $\tau=R C$, where $R$ is the resistance of the bulk liquid and $C$ is the double-layer capacitance. We find in our study that $\tau$ has an average value between 0.1 and $0.5 \mathrm{~ms}$ (Supporting Information), which is in the same range of the fast time scale measured with EIS, representing the diffusive charging mechanism. ${ }^{12}$ The measured time scales have a trend corresponding to the conductivity of the ionic liquids; the higher conductivity liquids have a shorter time constant, as expected. ${ }^{40}$ We do not observe the slow time scale $(O$ (seconds $))$ of the EIS experiments, which is likely caused by the roughness of our (polycrystalline) electrode surface. This slow process is interpreted as originating from ion reorientation, which occurs at the level of the compact ion layers on the electrode rather than the diffuse layer; therefore, it is more pronounced on smooth/crystalline electrodes and is most likely suppressed on polycrystalline platinum. ${ }^{12,31}$

The potential-dependent surface charge densities, $\sigma$, of the studied ionic liquids on platinum (Figure 1b) are the results of the cumulative summation of the charge density at each potential step, that is, $\sigma=\sum_{i} a_{i} \tau_{i}\left(1-\exp \left(-t^{\prime} / \tau_{i}\right)\right) \approx \sum_{i} a_{i} \tau_{i}$, where $a_{i}$ and $\tau_{i}$ are obtained from $\int_{0}^{t \prime} j \mathrm{~d} t\left(t^{\prime}=100 \mathrm{~ms}\right)$ of the $i$ th potential step. The differential capacitance per unit area, $C$, then can be calculated directly by differentiating the surface charge density with respect to the electrode potential, following the definition ${ }^{39}$

$$
C=\left(\frac{\partial \sigma}{\partial V}\right)_{T, \mu}
$$



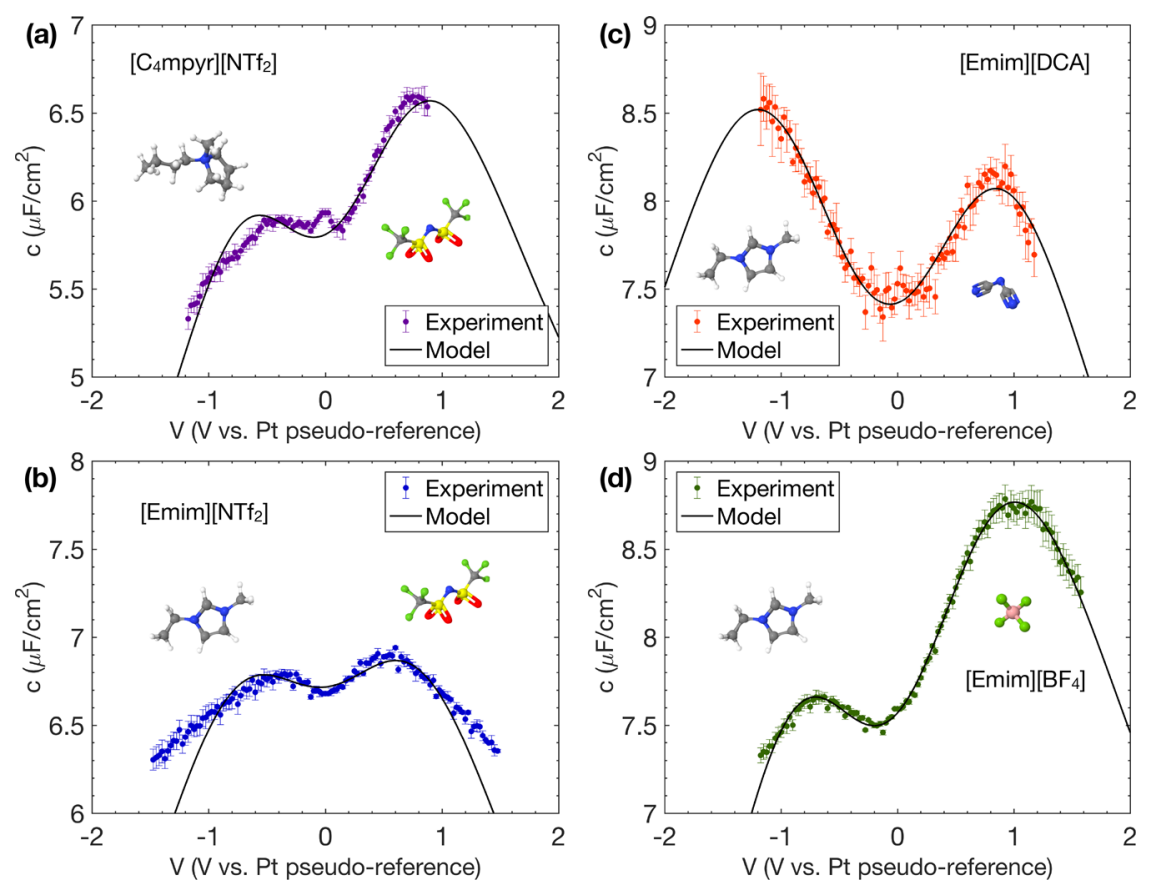

Figure 2. (a-d) Differential capacitance of pure ionic liquids $\left[\mathrm{C}_{4} \mathrm{mpyr}\right]^{+}\left[\mathrm{NTf}_{2}\right]^{-},[\mathrm{Emim}]^{+}\left[\mathrm{NTf}_{2}\right]^{-},\left[\mathrm{Emim}^{+}\left[\mathrm{BF}_{4}\right]^{-}\right.$, and $\left[\mathrm{Emim}^{+}[\mathrm{DCA}]^{-}\right.$, respectively, with the solid lines representing the fits from the extended mean-field model.

where $V$ is the electrode potential, $T$ is the temperature, and $\mu$ is the chemical potential.

The resulting capacitance curves are then fitted with the mean-field model of Goodwin-Kornyshev, where the shortrange ion correlations are taken into account, which can be written as

$$
C=\tilde{C}_{0} \frac{\cosh \left(\alpha u_{0} / 2\right)}{1+2 \gamma \sinh ^{2}\left(\alpha u_{0} / 2\right)} \sqrt{\frac{2 \gamma \sinh ^{2}\left(\alpha u_{0} / 2\right)}{\ln \left[1+2 \gamma \sinh ^{2}\left(\alpha u_{0} / 2\right)\right]}}
$$

where $\tilde{C}_{0}$ is a rescaled Debye capacitance, $\alpha$ is a rescaled parameter accounting for ion correlations, having values $<1(\alpha$ $=1$ recovers the case of zero short-range ion correlations), $u_{0}=$ $\mathrm{eV} / k_{\mathrm{B}} T$, and $\gamma$ is an ion fraction defined as the ratio of the total number of ions to the total number of sites available for them. The reader can find the detailed derivation of the model in the original paper of Goodwin et al. ${ }^{13,14}$ In the asymmetric case where the cation and anion are not of equivalent geometry, $\gamma$ is defined as follows ${ }^{7,13}$

$$
\gamma=\gamma_{-}+\frac{\gamma_{+}-\gamma_{-}}{1+\exp \left(\alpha u_{0} / 2\right)}
$$

where $\gamma_{+}$and $\gamma_{-}$are the ion fractions for cations and anions, respectively.

The capacitances of the ionic liquids $\left[\mathrm{C}_{4} \mathrm{mpyr}\right]^{+}\left[\mathrm{NTf}_{2}\right]^{-}$, $[\mathrm{Emim}]^{+}\left[\mathrm{NTf}_{2}\right]^{-},[\mathrm{Emim}]^{+}\left[\mathrm{BF}_{4}\right]^{-}$, and $[\mathrm{Emim}]^{+}[\mathrm{DCA}]^{-}$are plotted in Figure 2, together with the fits obtained from eq 2. Clearly, we see the capacitance curves reveal the predicted camel-shape behavior and the model fits our experimental data well.

The rescaled parameter, $\alpha$, in pure ionic liquids is found to have the value of $\sim 0.06$, which is far from 1 expected for the case of no ion-ion correlation, indicating that ion-ion repulsion dominates ion-pairing in ionic liquids. However, we cannot compare this result with other measurements because, to the best of our knowledge, this is the first attempt to extract this model parameter from experimental data. The ion fractions $\left(\gamma_{+}, \gamma_{-}\right)$for an asymmetric ionic liquids have been obtained from the fit, with values in the typically predicted range of 0.4 to 0.1 (Supporting Information). These doublehumped capacitance curves, which are different from the classical Gouy-Chapman theory, have been previously measured in the literature with the EIS technique but are often hidden in a "forest of peaks" and rarely fit the theory well. ${ }^{9,12,16,25,31}$ The qualitative agreement of our experimental data with the theoretical model and the quantitative agreement with many experimental observations from the EIS technique, supports the validity of our approach.

We note that the model shows better agreement in the liquids with molecules of smaller size and more simple shape (Figure $2 \mathrm{c}, \mathrm{d}$ ) than in the liquids having larger and more complex molecular geometries (Figure $2 \mathrm{a}, \mathrm{b}$ ). In the particular case of $[\mathrm{Emim}]^{+}\left[\mathrm{BF}_{4}\right]^{-}$, which has simplest molecular shapes among the liquids studied, the anions are spherical and substantially smaller than the cations, leading to a higher and steeper capacitance peak at positive polarization than at negative polarization. This behavior is described as a higher local ion concentration in the anion-rich region due to smaller anion sizes. ${ }^{7,16,18}$ However, this semiempirical explanation fails for the other liquids whose molecules are much less spherical (an assumption of the model). ${ }^{13,14}$ It is clear that the meanfield theory is limited here insofar as it does not take into account specific molecular geometries. ${ }^{7,13,14}$

The Debye capacitance $\left(C_{0}\right)$, as described by the mean-field model, is related to the electrostatic screening length, a characteristic length scale defined in the classical theory as the Debye length, $\lambda_{\mathrm{D}}$, by the relation $C_{0}=\epsilon \epsilon_{0} / \lambda_{\mathrm{D}}$, where $\epsilon$ is the dielectric constant of the medium and $\epsilon_{0}$ is the permittivity of free space. By using the low-frequency bulk dielectric constant of the ionic liquids (Supporting Information) and the capacitance values at the PZC $\left(C_{\mathrm{PZC}}\right)$ from our measurements of $5.8,6.6,7.5$, and $7.5 \mu \mathrm{F} / \mathrm{cm}^{2}$, we find screening lengths of 



Figure 3. (a) Differential capacitance of $[\text { Emim }]^{+}\left[\mathrm{NTf}_{2}\right]^{-} / \mathrm{DMSO}$ mixtures at different concentrations showing a crossover from camel-shape curves in dense concentrations to U-like curves in dilute regime. $(\mathrm{b}-\mathrm{e})$ Differential capacitance plotted together with the fits from the mean-field model at varied concentrations.

about $1.9,1.6,1.3$, and $1.5 \mathrm{~nm}$ for $\left[\mathrm{C}_{4} \mathrm{mpyr}\right]^{+}\left[\mathrm{NTf}_{2}\right]^{-}$, $[\mathrm{Emim}]^{+}\left[\mathrm{NTf}_{2}\right]^{-},[\mathrm{Emim}]^{+}[\mathrm{DCA}]^{-}$, and $[\mathrm{Emim}]^{+}\left[\mathrm{BF}_{4}\right]^{-}$, respectively. These screening lengths are $\sim 30$ times larger than those calculated using classical definition of the Debye length, and are about 10 times larger than the rescaled screening length, $\tilde{\lambda}_{\mathrm{D}}=\lambda_{\mathrm{D}} / \sqrt{ } \alpha$, calculated using the rescaled factor $(\alpha)$ obtained from the experimental data (Supporting Information). Long screening lengths in pure ionic liquids have been observed in force measurements and are explained as being caused by a low concentration of effective free ions in the liquids. ${ }^{41-44}$ However, the extent of ion pairing and dissociation is currently still a highly debated topic, and the final conclusion is not yet clear. This, in turn, affects physical interpretation of the fitting parameter, $\alpha$, whose value is directly related to ion correlations. ${ }^{13,14}$ Therefore, this interpretation may not be the case for our findings.

It must also be emphasized here that the measured capacitance is technically the total capacitance, that is, the combination of the Stern/compact layer and diffuse layer capacitances. As a result, the values of the measured capacitance at the PZC cannot be compared directly to the Debye capacitance, $C_{0}$, of the mean-field model without the knowledge of the compact layer capacitance. It could be that the capacitance measured in pure ionic liquids has a more significant contribution from the Stern capacitance rather than the Debye capacitance. The formation of liquid layers next to the charged surfaces is supported by X-ray reflectivity, ${ }^{45}$ atomic force microscopy (AFM), ${ }^{46}$ and surface force apparatus (SFA) experiments, ${ }^{47}$ but the layer-by-layer co/counterion densities are unknown.

Now that we have validated our experimental approach, we move on to study the differential capacitance in dilute ionic liquid by adding the solvent dimethyl sulfoxide (DMSO) to the ionic liquid $[\mathrm{Emim}]^{+}\left[\mathrm{NTf}_{2}\right]^{-}$, which forms miscible mixtures in a full range of the studied concentrations. The same CA procedure is applied for measuring the differential capacitance, and the results are shown in Figure 3. By varying the composition of the mixtures, we observe a clear transition from the pure to dilute regimes, as can be seen by a crossover of the capacitance curves from a camel shape to a U-like shape (Figure 3a), as predicted by the mean-field models. ${ }^{7,13,14}$ This crossover occurs between the concentration of 1 and $0.01 \mathrm{M}$, where the slope of the capacitance is steeper in the lower concentration mixtures, indicating a classical dilute electrolyte character. The mean-field model fitted to the experimental data shows good agreement (Figure $3 b-e)$, especially within $\pm(0.5$ to 1) V potential window from the PZC (Supporting Information), indicating the validity of the model and the importance of the short-range interaction effect added to it. The trend of the ion fraction $(\gamma)$ values is found to decrease (from $\sim 0.4$ to $\sim 0.1$ ) when more solvent is added to the ionic liquid (Supporting Information), resulting from the decrease in ion concentration. The parameter $\alpha$ is found to increase as the ion concentration decreases, as expected when the ion correlations are diminished by dilution.

The capacitance at the PZC is found to change nonmonotonically with concentration (Supporting Information). However, calculating $\lambda_{\mathrm{D}}$ from $C_{\mathrm{PZC}}$ without knowledge of the compact layer capacitance leads to discrepancies between $\lambda_{\mathrm{D}}$ measured here and the screening length from surface force measurement (Supporting Information).

In conclusion, we measured the differential capacitance of solvent-free and dilute ionic liquids using the CA technique and we find quantitative agreement between our direct capacitance measurement and the extended mean-field model of Goodwin-Kornyshev and qualitative agreement with several of the EIS experiments. Our measurements are a good indication of the validity of the model. The capacitances measured in the pure ionic liquids in our study show camelshape curves, as predicted by the models for moderate-packing ionic liquids, and the capacitances at the PZC are likely to relate to the compact layer capacitance rather than the diffuse layer capacitance.

Furthermore, crossover from camel to U-like capacitance curves is observed when solvent is added to the ionic liquids. A nonmonotonic dependence of the capacitance at the PZC with the concentration is found, similar to another independent study. ${ }^{48}$ However, quantitative conclusion cannot be made based only on the mean-field model due to lack of knowledge of the compact layer capacitance, which requires further theoretical studies or simulations.

Our observations provide important information for a wide range of uses, from theoretical model improvement to practical/technical implementation. In particular, we see relevance in the development of energy-storage device applications, where the energy density and power density have to be optimized.

The situation is expected to be more complicated in the case of nonmetallic electrodes, such as glassy carbon, graphite, or graphene, where the density of states (DOF) of the electrode material is limited and the electrode capacitance can have a dramatic effect on the measured capacitance, ${ }^{48-51}$ but for 
metal electrodes, direct application of the Goodwin-Kornyshev analytical model is satisfactory.

\section{ASSOCIATED CONTENT}

\section{S Supporting Information}

The Supporting Information is available free of charge on the ACS Publications website at DOI: 10.1021/acs.jpclett.7b02946.

Data analysis method; dielectric constants, conductivity, and the characteristic time constants of pure ionic liquids; fitting parameters obtained from capacitance data and calculated Debye lengths in pure and dilute ionic liquids; capacitance plots at different ionic liquid concentrations; comparison of our data with SFA results; the electrochemical windows of ionic liquids and experimental evidence of the effect of open circuit potential on the reproducibility. (PDF)

\section{AUTHOR INFORMATION}

\section{Corresponding Author}

*E-mail: j.r.t.seddon@utwente.nl.

\section{ORCID 8}

James R. T. Seddon: 0000-0002-6069-3879

\section{Notes}

The authors declare no competing financial interest.

\section{ACKNOWLEDGMENTS}

We thank Serge Lemay for useful discussions on the electrochemical techniques. We also acknowledge Jelle Schoppink and Sytze Bakker for their hard work on the preliminary measurements. M.J. acknowledges The Development and Promotion of Science and Technology Talents project for the financial support.

\section{REFERENCES}

(1) Welton, T. Room-Temperature Ionic Liquids. Solvents for Synthesis and Catalysis. Chem. Rev. 1999, 99, 2071-2084.

(2) Ohno, H. Electrochemical Aspects of Ionic Liquids; John Wiley \& Sons, 2005.

(3) Armand, M.; Endres, F.; MacFarlane, D. R.; Ohno, H.; Scrosati, B. Ionic-Liquid Materials for the Electrochemical Challenges of the Future. Nat. Mater. 2009, 8, 621-629.

(4) Kim, T. Y.; Lee, H. W.; Stoller, M.; Dreyer, D. R.; Bielawski, C. W.; Ruoff, R. S.; Suh, K. S. High-Performance Supercapacitors based on Poly (Ionic Liquid)-Modified Graphene Electrodes. ACS Nano 2011, 5, 436-442.

(5) Barrosse-Antle, L.; Bond, A.; Compton, R.; O'Mahony, A.; Rogers, E.; Silvester, D. Voltammetry in Room Temperature Ionic Liquids: Comparisons and Contrasts with Conventional Electrochemical Solvents. Chem. - Asian J. 2010, 5, 202-230.

(6) Fedorov, M. V.; Kornyshev, A. A. Ionic Liquids at Electrified Interfaces. Chem. Rev. 2014, 114, 2978-3036.

(7) Kornyshev, A. A. Double-Layer in Ionic Liquids: Paradigm Change? J. Phys. Chem. B 2007, 111, 5545.

(8) Lockett, V.; Sedev, R.; Ralston, J.; Horne, M.; Rodopoulos, T. Differential Capacitance of the Electrical Double Layer in Imidazolium-Based Ionic Liquids: Influence of Potential, Cation Size, and Temperature. J. Phys. Chem. C 2008, 112, 7486-7495.

(9) Lockett, V.; Horne, M.; Sedev, R.; Rodopoulos, T.; Ralston, J. Differential Capacitance of the Double Layer at the Electrode/Ionic Liquids Interface. Phys. Chem. Chem. Phys. 2010, 12, 12499-12512.

(10) Zheng, J.; Goonetilleke, P.; Pettit, C.; Roy, D. Probing the Electrochemical Double Layer of an Ionic Liquid using Voltammetry and Impedance Spectroscopy: A Comparative Study of Carbon
Nanotube and Glassy Carbon Electrodes in [EMIM]+[EtSO 4]-. Talanta 2010, 81, 1045-1055.

(11) Zheng, J.; Moganty, S. S.; Goonetilleke, P. C.; Baltus, R. E.; Roy, D. A Comparative Study of the Electrochemical Characteristics of $[\mathrm{Emim}+][\mathrm{BF} 4-]$ and $[\mathrm{Bmim}+][\mathrm{BF} 4-]$ Ionic Liquids at the Surfaces of Carbon Nanotube and Glassy Carbon Electrodes. J. Phys. Chem. C 2011, 115, 7527-7537.

(12) Roling, B.; Drüschler, M.; Huber, B. Slow and Fast Capacitive Process Taking Place at the Ionic Liquid/Electrode Interface. Faraday Discuss. 2012, 154, 303-311.

(13) Goodwin, Z. A.; Feng, G.; Kornyshev, A. A. Mean-Field Theory of Electrical Double Layer In Ionic Liquids with Account of ShortRange Correlations. Electrochim. Acta 2017, 225, 190-197.

(14) Goodwin, Z. A.; Kornyshev, A. A. Underscreening, Overscreening and Double-Layer Capacitance. Electrochem. Commun. 2017, 82, 129-133.

(15) Oldham, K. B. A Gouy-Chapman-Stern Model of the Double Layer at a (Metal)/(Ionic Liquid) Interface. J. Electroanal. Chem. 2008, 613, 131-138.

(16) Fedorov, M. V.; Georgi, N.; Kornyshev, A. A. Double Layer in Ionic Liquids: The Nature of the Camel Shape of Capacitance. Electrochem. Commun. 2010, 12, 296-299.

(17) Trulsson, M.; Algotsson, J.; Forsman, J.; Woodward, C. E. Differential Capacitance of Room Temperature Ionic Liquids: The Role of Dispersion Forces. J. Phys. Chem. Lett. 2010, 1, 1191-1195.

(18) Georgi, N.; Kornyshev, A. A.; Fedorov, M. V. The Anatomy of the Double Layer and Capacitance in Ionic Liquids with Anisotropic Ions: Electrostriction vs. Lattice Saturation. J. Electroanal. Chem. 2010, 649, 261-267.

(19) Alam, M. T.; Islam, M. M.; Okajima, T.; Ohsaka, T. Measurements of Differential Capacitance at Mercury/RoomTemperature Ionic Liquids Interfaces. J. Phys. Chem. C 2007, 111, 18326-18333.

(20) Alam, M. T.; Islam, M. M.; Okajima, T.; Ohsaka, T. Measurements of Differential Capacitance in Room Temperature Ionic Liquid at Mercury, Glassy Carbon and Gold Electrode Interfaces. Electrochem. Commun. 2007, 9, 2370-2374.

(21) Alam, M. T.; Islam, M. M.; Okajima, T.; Ohsaka, T. Ionic Liquid Structure Dependent Electrical Double Layer at the Mercury Interface. J. Phys. Chem. C 2008, 112, 2601-2606.

(22) Alam, M. T.; Islam, M. M.; Okajima, T.; Ohsaka, T. Capacitance Measurements in a Series of Room-temperature Ionic Liquids at Glassy Carbon and Gold Electrode Interfaces. J. Phys. Chem. C 2008, 112, 16600-16608.

(23) Alam, M. T.; Islam, M. M.; Okajima, T.; Ohsaka, T. Electrical Double Layer in Mixtures of Room-Temperature Ionic Liquids. J. Phys. Chem. C 2009, 113, 6596-6601.

(24) Alam, M. T.; Masud, J.; Islam, M. M.; Okajima, T.; Ohsaka, T. Differential Capacitance at Au (111) in 1-alkyl-3-methylimidazolium tetrafluoroborate based Room-Temperature Ionic Liquids. J. Phys. Chem. C 2011, 115, 19797-19804.

(25) Islam, M. M.; Alam, M. T.; Ohsaka, T. Electrical Double-layer Structure in Ionic Liquids: A Corroboration of the Theoretical Model by Experimental Results. J. Phys. Chem. C 2008, 112, 16568-16574.

(26) Islam, M. M.; Alam, M. T.; Okajima, T.; Ohsaka, T. Electrical Double Layer Structure in Ionic Liquids: An Understanding of the Unusual Capacitance-Potential Curve at a Nonmetallic Electrode. J. Phys. Chem. C 2009, 113, 3386-3389.

(27) Gnahm, M.; Pajkossy, T.; Kolb, D. The Interface between Au (111) and an Ionic Liquid. Electrochim. Acta 2010, 55, 6212-6217.

(28) Drüschler, M.; Huber, B.; Roling, B. On Capacitive Processes at the Interface between 1-Ethyl-3-methylimidazolium tris (pentafluoroethyl) trifluorophosphate and $\mathrm{Au}$ (111). J. Phys. Chem. C 2011, 115, 6802-6808.

(29) Pajkossy, T.; Kolb, D. M. The Interfacial Capacitance of Au (100) in an Ionic Liquid, 1-butyl-3-methyl-imidazolium hexafluorophosphate. Electrochem. Commun. 2011, 13, 284-286.

(30) Atkin, R.; Borisenko, N.; Drüschler, M.; El Abedin, S. Z.; Endres, F.; Hayes, R.; Huber, B.; Roling, B. An in situ STM/AFM and 
Impedance Spectroscopy Study of the Extremely Pure 1-butyl-1methylpyrrolidinium tris (pentafluoroethyl) trifluorophosphate/Au (111) Interface: Potential Dependent Solvation Layers and the Herringbone Reconstruction. Phys. Chem. Chem. Phys. 2011, 13, 6849-6857.

(31) Atkin, R.; Borisenko, N.; Drüschler, M.; Endres, F.; Hayes, R.; Huber, B.; Roling, B. Structure and Dynamics of the Interfacial Layer between Ionic Liquids and Electrode Materials. J. Mol. Liq. 2014, 192, 44-54.

(32) Li, M.-G.; Chen, L.; Zhong, Y.-X.; Chen, Z.-B.; Yan, J.-W.; Mao, B.-W. The Electrochemical Interface of Ag (111) in 1-ethyl-3methylimidazolium bis (trifluoromethylsulfonyl) imide Ionic Liquid-A Combined in-situ Scanning Probe Microscopy and Impedance Study. Electrochim. Acta 2016, 197, 282-289.

(33) Roling, B.; Drüschler, M. Comments on "Intrinsic Limitations of Impedance Measurements in Determining Electric Double Layer Capacitances" by H. Wang and L. Pilon [Electrochim. Acta 63 (2012) 55]. Electrochim. Acta 2012, 76, 526-528.

(34) Wang, H.; Pilon, L. Intrinsic Limitations of Impedance Measurements in Determining Electric Double Layer Capacitances. Electrochim. Acta 2012, 63, 55-63.

(35) Wang, H.; Pilon, L. Reply to Comments on "Intrinsic Limitations of Impedance Measurements in Determining Electric Double Layer Capacitances" by H. Wang, L. Pilon [Electrochimica Acta 63 (2012) 55]. Electrochim. Acta 2012, 76, 529-531.

(36) Drüschler, M.; Roling, B. Commentary on 'The Interface between $\mathrm{Au}$ (111) and an Ionic Liquid. Electrochim. Acta 2011, 56, $7243-7245$.

(37) Pajkossy, T. Response to the Commentary of Marcel Drüschler and Bernhard Roling on 'The Interface between $\mathrm{Au}$ (111) and an Ionic Liquid. Electrochim. Acta 2011, 56, 7246-7247.

(38) Zhou, W.; Inoue, S.; Iwahashi, T.; Kanai, K.; Seki, K.; Miyamae, T.; Kim, D.; Katayama, Y.; Ouchi, Y. Double Layer Structure and Adsorption/Desorption Hysteresis of Neat Ionic Liquid on $\mathrm{Pt}$ Electrode Surface-An in-situ IR-Visible Sum-Frequency Generation Spectroscopic Study. Electrochem. Commun. 2010, 12, 672-675.

(39) Bard, A. J.; Faulkner, L. R. Electrochemical Methods: Fundamentals and Applications, 2nd ed.; John Wiley \& Son, 2001.

(40) Chaban, V. V.; Voroshylova, I. V.; Kalugin, O. N.; Prezhdo, O. V. Acetonitrile Boosts Conductivity of Imidazolium Ionic Liquids. J. Phys. Chem. B 2012, 116, 7719-7727.

(41) Gebbie, M. A.; Valtiner, M.; Banquy, X.; Fox, E. T.; Henderson, W. A.; Israelachvili, J. N. Ionic Liquids Behave as Dilute Electrolyte Solutions. Proc. Natl. Acad. Sci. U. S. A. 2013, 110, 9674-9679.

(42) Gebbie, M. A.; Dobbs, H. A.; Valtiner, M.; Israelachvili, J. N. Long-range Electrostatic Screening in Ionic Liquids. Proc. Natl. Acad. Sci. U. S. A. 2015, 112, 7432-7437.

(43) Gebbie, M. A.; Smith, A. M.; Dobbs, H. A.; Lee, A. A.; Warr, G. G.; Banquy, X.; Valtiner, M.; Rutland, M. W.; Israelachvili, J. N.; Perkin, S.; Atkin, R. Long Range Electrostatic Forces in Ionic Liquids. Chem. Commun. 2017, 53, 1214-1224.

(44) Smith, A. M.; Lee, A. A.; Perkin, S. The Electrostatic Screening Length in Concentrated Electrolytes Increases with Concentration. J. Phys. Chem. Lett. 2016, 7, 2157-2163.

(45) Mezger, M.; Ocko, B. M.; Reichert, H.; Deutsch, M. Surface Layering and Melting in an Ionic Liquid Studied by Resonant Soft Xray Reflectivity. Proc. Natl. Acad. Sci. U. S. A. 2013, 110, 3733-3737.

(46) Hayes, R.; Borisenko, N.; Tam, M. K.; Howlett, P. C.; Endres, F.; Atkin, R. Double Layer Structure of Ionic Liquids at the Au (111) Electrode Interface: An Atomic Force Microscopy Investigation. J. Phys. Chem. C 2011, 115, 6855-6863.

(47) Perkin, S. Ionic Liquids in Confined Geometries. Phys. Chem. Chem. Phys. 2012, 14, 5052-5062.

(48) Bozym, D. J.; Uralcan, B.; Limmer, D. T.; Pope, M. A.; Szamreta, N. J.; Debenedetti, P. G.; Aksay, I. A. Anomalous Capacitance Maximum of the Glassy Carbon-Ionic Liquid Interface through Dilution with Organic Solvents. J. Phys. Chem. Lett. 2015, 6, $2644-2648$
(49) Gerischer, H. An Interpretation of the Double Layer Capacity of Graphite Electrodes in Relation to the Density of States at the Fermi Level. J. Phys. Chem. 1985, 89, 4249-4251.

(50) Gerischer, H.; McIntyre, R.; Scherson, D.; Storck, W. Density of the Electronic States of Graphite: Derivation from Differential Capacitance Measurements. J. Phys. Chem. 1987, 91, 1930-1935.

(51) Kornyshev, A.; Luque, N.; Schmickler, W. Differential Capacitance of Ionic Liquid Interface with Graphite: The Story of Two Double Layers. J. Solid State Electrochem. 2014, 18, 1345-1349. 